\title{
Autopercepción del peso a través de figuras corporales en niños en edad escolar de Piedras Negras, Coahuila, México
}

\author{
Felipe J. Uribe-Salas ${ }^{1 *}$, María del Carmen Portillo-Téllez ${ }^{1}$, Juan Parra-Ávila ${ }^{1}$, Suzel Hernández-Corral², \\ Regina Alonso-Tovar ${ }^{1}$, María Soledad Acevedo-Casillas ${ }^{3}$ y Juan Felipe-Mayo ${ }^{1}$ \\ ${ }^{1}$ El Colegio de la Frontera Norte, Tijuana, Baja California; ${ }^{2}$ Escuela de Ciencias de la Salud, Universidad Autónoma de Coahuila; ${ }^{3}$ Universidad \\ Vizcaya de las Américas, Campus Piedras Negras. Coahuila, México
}

\begin{abstract}
Resumen
Introducción: Ante la tendencia mundial en el incremento del índice de masa corporal (IMC) en todos los grupos de edad, se han buscado estrategias de control. La baja percepción en el exceso de peso en niños escolares es un tema pendiente de explorar en la frontera norte de México. Métodos: Se obtuvo el IMC de alumnos de tercero a sexto grado de educación básica. Se compararon los IMC, percibido e ideal, con el IMC medido, evaluando subestimaciones y sobrestimaciones con respecto a este último. Se calcularon las prevalencias de la exactitud en el IMC percibido y la discrepancia de la imagen ideal respecto al IMC. Resultados: Se estudiaron 155 niños (43.8\% del sexo femenino) con una edad promedio de 10.2 años. La prevalencia de la exactitud del IMC percibido respecto al medido fue del $59.4 \%$. La prevalencia de discrepancia entre el IMC ideal y el medido fue del 44.4\%. La comparación del IMC percibido con el medido mostró una sobrestimación en la categoría de peso bajo (33\%) y subestimaciones en las categorías de peso normal (12.4\%), sobrepeso (85\%) y obesidad (81.6\%). Al comparar el IMC ideal con el medido, se mostró una sobrestimación absoluta en la categoría de peso bajo y subestimaciones en las categorías de peso normal, sobrepeso y obesidad, del $5.6 \%, 100 \%$ y $97.4 \%$, respectivamente. Conclusiones: El presente trabajo muestra en forma consistente la subestimación que los escolares hacen de su peso, particularmente los clasificados en las categorías de sobrepeso y obesidad. en un contexto demográfico donde la prevalencia de ambas categorías en este estudio fue alta, del $38 \%$.
\end{abstract}

Palabras clave: Adolescentes. Obesidad. Sobrepeso. Percepción corporal. Autopercepción.

\section{Self-perception of weight in school-age children from Piedras Negras, Coahuila, Mexico}

\section{Abstract}

Background: The prevalence of overweight and obesity has increased worldwide. In this context, the lack of awareness of excess weight among overweight and obese children is a public health concern that needs to be explored in the Northern border of Mexico. Methods: Body mass index (BMI) was calculated from children from third to sixth grades of basic education. The Children's Body Image Scale was applied to evaluate perceived and ideal BMI comparing against calculated BMI. We also analyzed the frequency of accurate perceivers of actual weight and discrepancy of ideal weight against calculated BMI. Results: We studied 155 children (43.8\% were female) with a mean age of 10.2 years. Accuracy prevalence of

Correspondencia:

${ }^{*}$ Felipe J. Uribe-Salas

E-mail: fjuribe@ colef.mx
Fecha de recepción: 17-04-2018

Fecha de aceptación: 02-07-2018

DOI: 10.24875/BMHIM. 18000041
Disponible en internet: 01-11-2018 Bol Med Hosp Infant Mex. 2018;75:366-372 www.bmhim.com

1665-1146/@ 2018. Hospital Infantil de México Federico Gómez, impreso por Permanyer México SA de CV, todos los derechos reservados. 
BMI perceived respect to calculated BMI was $59.4 \%$. Discrepancy prevalence of ideal BMI respect to calculated BMI was $44.4 \%$. Comparison of perceived BMI respect to calculated BMI showed an overestimation on low weight category (33\%) and subestimations in categories of normal weight (12.4\%), overweight (85\%) and obesity (81.6\%). Comparison of ideal BMI respect to calculated BMI showed an absolute overestimation of low weight and underestimations in categories of normal weight, overweight and obesity of $5.6 \%, 100 \%$, and $97.4 \%$, respectively. Conclusions: The results of this work showed a consistent underestimation of children's body weight, particularly among those classified in categories of overweight and obesity in a demographic context in which the prevalence of both categories was of $38 \%$ in this study.

Key words: Adolescents. Obesity. Overweight. Body image. Self-concept.

\section{Introducción}

Se han documentado incrementos en las frecuencias de sobrepeso y obesidad en todos los grupos de edad en todo el mundo, y en forma particular en México ${ }^{1-3}$. Uno de los grupos de mayor relevancia para el establecimiento de medidas preventivas contra el incremento extremo de peso es la población en edad escolar ${ }^{4}$. En México, la Encuesta Nacional de Salud y Nutrición (ENSANUT) $2012^{5}$ mostró prevalencias conjuntas de sobrepeso y obesidad del $34.4 \%$ en niños entre 5 y 11 años de edad. La misma encuesta, pero de $2016^{3}$, mostró un ligero descenso en la magnitud conjunta de sobrepeso y obesidad, de 1.2 puntos porcentuales, con respecto al resultado de 2012 en niños entre 5 y 11 años de edad. Lo anterior podría ser indicativo de que las políticas y los programas nacionales de prevención y control de la obesidad analizados por Barquera-Cervera, et al. ${ }^{6}$ empiezan a tener efecto en México. No obstante, la magnitud conjunta de sobrepeso y obesidad sigue siendo alta, de al menos un tercio del total de niños.

La ciudad de Piedras Negras está situada en la frontera norte del Estado de Coahuila. Limita al norte con el municipio de Jiménez, al sur con los de Nava y Zaragoza, y al noreste y oeste con los EE.UU. Su superficie es de $914.20 \mathrm{~km}^{2}$ y está situada en las coordenadas $100^{\circ} 34^{\prime} 5^{\prime \prime}$ longitud oeste y $28^{\circ} 43^{\prime} 20^{\prime \prime}$ latitud norte ${ }^{7}$. En 2015 contaba con una población de 163,595 habitantes $^{8}$. Los resultados de la ENSANUT para la frontera norte de México mostraron una prevalencia conjunta de sobrepeso y obesidad del $33.9 \%$ en 2006 , y del $38 \%$ en la encuesta para Coahuila en 2012 para niños entre 5 y 11 años de edad ${ }^{9,10}$. Un estudio realizado en Piedras Negras en alumnos de tercer grado de educación primaria mostró una prevalencia conjunta de sobrepeso y obesidad del $38.3 \%{ }^{11}$. Estos resultados de prevalencias altas de sobrepeso y obesidad en niños de edad escolar en la frontera del norte de México sugieren la existencia de un contexto económico, social y cultural que puede estar teniendo impacto en la ganancia de peso en la infancia. Anaya y Al-Delaimy ${ }^{12}$ han planteado un efecto contextual de la región fronteriza entre México y los EE.UU. en la presencia de una desproporcionada mortalidad por enfermedades cardiovasculares, lo cual obliga a estudiar los fenómenos de salud en esta región.

La percepción y la insatisfacción con la imagen corporal han sido estudiadas ampliamente en adolescentes y adultos, pero poco en niños prepuberales ${ }^{13}$. Se ha reportado, sobre todo, que la magnitud de la insatisfacción con la imagen corporal se ha incrementado en niños prepuberales ${ }^{14,15}$, y que frecuentemente se acompaña de estrategias para bajar de peso y de trastornos de la conducta alimentaria ${ }^{16}$. Se ha observado que los niños con sobrepeso y obesidad tienden a representarse en figuras más delgadas que las que les corresponden, mostrando una insatisfacción con su imagen corporal real ${ }^{17}$. Para evaluar la percepción y la insatisfacción con la imagen corporal se han utilizado diferentes formatos de siluetas corporales que proporcionan una medida de la imagen percibida, la ideal y la real, esta última medida por el índice de masa corporal (IMC) $)^{18-20}$. No obstante, la relación entre el IMC de los niños y el diseño de las siluetas no se había establecido hasta que Truby y Paxton ${ }^{13}$ desarrollaron una plantilla con dibujos de figuras corporales basada en fotografías de niños y niñas con diferentes percentiles del IMC y con edades entre 8 y 12 años. En México se han utilizado las escalas de figuras corporales de Stunkard y de Keds, pero no las diseñadas por Truby y Paxton ${ }^{13}$. La de Stunkard ha sido aplicada por el personal de salud para identificar obesidad en lugares donde se dificulta la toma de medidas somatométricas para el cálculo del IMC en niños de edad escolar ${ }^{21}$. Los estudios realizados en niños de edad escolar para evaluar la percepción corporal han sido escasos. Un estudio realizado en una comunidad suburbana de Yucatán, en adolescentes entre 10 y 17 años, mostró una alta frecuencia de peso bajo del $26 \%$, mientras que la obesidad fue del $11.9 \%$. Cuando se evaluó la percepción corporal 
utilizando las figuras de Stunkard, se observó la subestimación del peso bajo y la sobrestimación del peso normal y de la obesidad ${ }^{22}$. Otros autores, que estudiaron alumnos entre 9 y 12 años de edad en un poblado del Estado de México, mostraron una alta frecuencia de peso bajo, del $62.6 \%$, peso normal en el $32.7 \%$ y sobrepeso en el $4.7 \%$. Al evaluar la percepción corporal con las siluetas de Stunkard, se observó una subestimación pequeña del peso bajo, del $2.8 \%$, mientras que el $83 \%$ se percibió más robusto de lo que en realidad era ${ }^{23}$. Estos resultados discrepan de lo reportado por la literatura internacional, en la que se señala que los niños tienden a subestimar sus dimensiones corporales cuando son extremas ${ }^{17,24-26}$. Aunque en la frontera norte del Estado de Coahuila se presenta una frecuencia alta de sobrepeso y obesidad en niños de edad escolar, no se ha evaluado el comportamiento de la autopercepción de la imagen corporal en esa población. El presente trabajo tuvo por objeto determinar si existen diferencias de medida entre la imagen corporal percibida, la ideal y la real, en población escolar de Piedras Negras.

\section{Métodos}

En el mes de junio de 2017 se inició un estudio de tipo transversal que incluyó alumnos de tercero a sexto grado en escuelas de educación primaria en la ciudad de Piedras Negras, Coahuila. Para este estudio se reportan las entrevistas realizadas a los alumnos de tercero a sexto grado de dos escuelas públicas. Para tener acceso al estudio de los niños y de sus padres o tutores, se diseñaron dos cartas de consentimiento informado: una para las autoridades de la institución escolar y otra para ser firmada por los padres o tutores de los niños. En ambas cartas se aseguraba la confidencialidad de la información obtenida. El protocolo del estudio fue sometido al Comité de Investigación de El Colegio de la Frontera Norte.

\section{Medidas antropométricas}

Se realizó una estandarización del personal para la toma de las mediciones somatométricas siguiendo el método ISAK ${ }^{27}$. Las mediciones se hicieron en los niños sin zapatos y con ropa ligera. Se procedió a tomar el peso de los niños con una báscula (Seca 803) con una precisión de $0.1 \mathrm{~kg}$. Para la talla se utilizó un estadímetro (Seca 213) con una precisión en las tomas de $0.1 \mathrm{~cm}$.

\section{Percepción de la imagen corporal}

Se utilizó la Escala de Imagen Corporal para Niños (CBIS, por su acrónimo en inglés), compuesta por siete figuras basadas en fotografías de niños y niñas con diferentes percentiles $(3,10,25,50,75,90$ y 97$)$ de IMC, de tal manera que permite recodificar los valores para construir cuatro categorías: peso bajo (percentil 3), normal (percentiles 10 a 75), sobrepeso (percentil 90) y obesidad (percentil 97), de acuerdo con la clasificación de los Centers for Disease Control and Prevention $(C D C)^{13}$. Dicha escala fue evaluada en su reproducibilidad para ser utilizada con estándares internacionales ${ }^{28}$. Para evaluar la percepción del peso de los niños, se les pidió que seleccionaran en la escala de figuras aquella que representaba su volumen corporal actual, a lo que se llamó IMC percibido. También se pidió, en una segunda pregunta, que seleccionaran la figura corporal ideal, es decir, aquella que les gustaría tener a los niños, y esta se llamó IMC ideal.

\section{Cálculo de los percentiles del IMC}

Para este cálculo se utilizó el programa en Excel completado por Romero ${ }^{29}$. En dicho programa pueden calcularse los percentiles de peso, talla e IMC estratificados por sexo y edad (entre 2 y 20 años). El programa está organizado para trabajar con las curvas de crecimiento diseñadas por los CDC. Se utilizaron las siguientes categorías del IMC calculado: bajo peso (por debajo del percentil 5); peso normal (entre los percentiles 5 y 85); sobrepeso (entre los percentiles 85 y 95); y obesidad (por arriba del percentil 95). Este cálculo fue definido como IMC medido.

\section{Plan de análisis}

Se realizaron estadísticas descriptivas por sexo, edad y grado escolar. Se estudió la relación entre las categorías del IMC medido y del IMC percibido, a fin de evaluar la exactitud en la percepción de la propia imagen corporal ${ }^{13}$. Un procedimiento similar se realizó entre las categorías de los IMC medido e ideal para evaluar la satisfacción de los niños con su propia imagen corporal ${ }^{13}$. Para evaluar la concordancia entre las categorías del IMC medido y las del IMC percibido, se calculó el índice de Kappa ponderado de acuerdo con el método propuesto por Zklo y Nieto ${ }^{30}$. Para cumplir con el objetivo de este estudio, se evaluaron la exactitud del IMC percibido y la discrepancia del IMC ideal en su relación con las variables sexo, edad e IMC 
Tabla 1. Relación entre el índice de masa corporal medido y el índice de masa corporal percibido por escolares en Piedras Negras, Coahuila, México, 2017

\begin{tabular}{|c|c|c|c|c|c|}
\hline \multirow[t]{2}{*}{ IMC percibido } & \multicolumn{4}{|c|}{ IMC medido } & \multirow[t]{2}{*}{ Total } \\
\hline & Bajo peso, n (\%) & Peso normal, n (\%) & Sobrepeso, n (\%) & Obesidad, n (\%) & \\
\hline Bajo peso & $4(66.7)$ & $11(12.4)$ & 0 & $2(5.3)$ & 17 \\
\hline Peso normal & $2(33.3)$ & 77 (86.5) & $17(85.0)$ & $22(57.9)$ & 118 \\
\hline Sobrepeso & 0 & $1(1.1)$ & $3(15.0)$ & $7((18.4)$ & 11 \\
\hline Obesidad & 0 & 0 & 0 & $7(18.4)$ & 7 \\
\hline Total & 6 & 89 & 20 & 38 & $153^{*}$ \\
\hline
\end{tabular}

*Índice de Kappa ponderado $=0.30$.

IMC: índice de masa corporal.

medido de los escolares analizados. Para evaluar el tamaño del efecto de la relación entre las variables dependientes e independientes se utilizó la prueba $\mathrm{V}$ de Cramer. El análisis se realizó con el programa estadístico SPSS versión 19.

\section{Resultados}

Se estudiaron, en total, 155 niños, de los cuales un poco menos de la mitad eran de sexo femenino (43.8\%). La edad promedio de los entrevistados fue de 10.23 años (mediana de 10 y varianza de 1.69 , recorrido de 8 a 13 años), teniendo la mayor frecuencia en 11 años (27.9\%), seguida de niños con 9 y 10 años $(24.5 \%$ y $18.8 \%$, respectivamente). La distribución de los niños por grado escolar fue mayor en los grupos de quinto y sexto $(29.7 \%$ y $29.0 \%$, respectivamente). Los grupos de tercero y cuarto grado tuvieron una magnitud porcentual similar (20.6\%).

La tabla 1 muestra la distribución de los resultados del IMC medido en las columnas y de los resultados del IMC percibido en los renglones. Se observa que cuatro de seis niños con bajo peso (66.7\%) identificaron con exactitud la imagen que les correspondía según su IMC. La mayor exactitud en la selección de la imagen corporal se presentó en los niños clasificados con peso normal según su IMC medido, ya que 77 de 86 niños seleccionaron la imagen corporal que les correspondía, dando una exactitud del $86.5 \%$. De 20 niños con sobrepeso, solo tres seleccionaron la imagen corporal que les correspondía, dando una exactitud del $15 \%$. Asimismo, de 38 niños con obesidad, solo siete seleccionaron las figuras corporales correspondientes a su IMC medido, dando una exactitud del $18.4 \%$. En total, 91 niños seleccionaron correctamente las figuras corporales actuales que les correspondían de acuerdo con su IMC medido, dando una exactitud en la percepción de la imagen corporal del $59.4 \%$, lo cual se refleja en un coeficiente de Kappa ponderado de 0.30.

En la tabla 2 se muestra la distribución de los resultados del IMC medido en las columnas, y la de los resultados del IMC ideal en los renglones. Puede observarse que ninguno de los niños con bajo peso seleccionó la imagen corporal que le correspondía, presentando una discrepancia absoluta en esa categoría. La menor discrepancia se presentó en los niños clasificados en la categoría de peso normal, pues cinco de $89(5.6 \%)$ discreparon con su propia imagen corporal como ideal. Sin embargo, los niños con sobrepeso tuvieron una discrepancia absoluta al seleccionar imágenes más delgadas, particularmente las de peso normal y de Bajo peso. De 30 individuos con obesidad, solo uno (2.6\%) seleccionó correctamente la figura corporal que le correspondía, pues la mayoría seleccionaron imágenes más delgadas, particularmente las de peso normal. Del total, 68 individuos discreparon con la imagen corporal que les correspondía, dando una insatisfacción con el peso corporal del $44.4 \%$.

Finalmente, la tabla 3 muestra las frecuencias absolutas y relativas de la exactitud del IMC percibido y de las discrepancias entre el IMC ideal con respecto al IMC medido en ambos casos. Puede observarse que ninguna de las variables dependientes tuvo relación significativa con el sexo y la edad, pero sí con el IMC medido. Las categorías del IMC medido en las que se realizaron mayores percepciones correctas fueron en el peso normal ( $86.5 \%$ ) y el bajo peso $(66.7 \%)$, mientras que hubo frecuencias menores de selección correcta en el sobrepeso (15\%) y la obesidad (18.4\%) (V de Cramer 0.67; $p<0.001)$. En cuanto a las discrepancias 
Tabla 2. Relación entre el índice de masa corporal medido y el índice de masa corporal ideal reportado por escolares en Piedras Negras, Coahuila, México, 2017

\begin{tabular}{|c|c|c|c|c|c|}
\hline \multirow[t]{2}{*}{ IMC ideal } & \multicolumn{4}{|c|}{ IMC medido } & \multirow[t]{2}{*}{ Total } \\
\hline & Bajo peso, n (\%) & Peso normal, n (\%) & Sobrepeso, n (\%) & Obesidad, n (\%) & \\
\hline Bajo peso & 0 & $5(5.6)$ & $6(30.0)$ & $4(10.5)$ & 15 \\
\hline Peso normal & $5(83.3)$ & $84(94.4)$ & $14(70.0)$ & 31 (81.6) & 134 \\
\hline Sobrepeso & $1(16.7)$ & 0 & 0 & $2((5.3)$ & 3 \\
\hline Obesidad & 0 & 0 & 0 & $1(2.6)$ & 1 \\
\hline Total & 6 & 89 & 20 & 38 & 153 \\
\hline
\end{tabular}

IMC: índice de masa corporal.

Tabla 3. Diferencias en el índice de masa corporal percibido, ideal y medido, por sexo y edad, de escolares en Piedras Negras, Coahuila, México, 2017

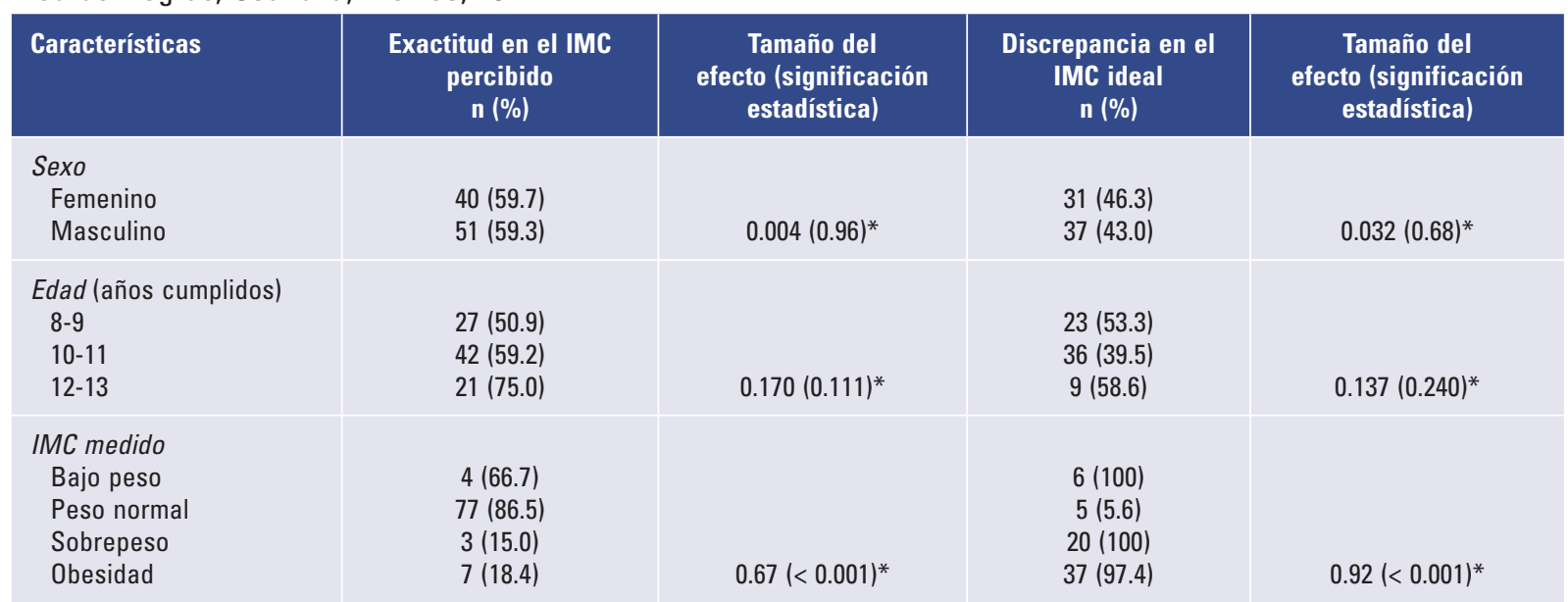

*Prueba V de Cramer.

IMC: índice de masa corporal.

entre el IMC ideal y el IMC medido, se encontró que todos los individuos en las categorías de bajo peso y sobrepeso, y casi todos en la categoría de obesidad (97.4\%), no seleccionaron esas figuras. La categoría de menor discrepancia fue la de peso normal (5.6\%) (V de Cramer 0.92; $p<0.001$ ).

\section{Discusión}

La utilización de la CBIS presenta la ventaja de que fue diseñada tomando en cuenta las características corporales de los niños entre 7 y 12 años de edad, y además fue evaluada en su reproducibilidad y utilizada con los estándares internacionales del cálculo del $\mathrm{IMC}^{27}$. Se ha mostrado, además, que el grado de precisión en que los niños perciben su imagen corporal es mayor al utilizar la CBIS, en comparación con un instrumento que pide respuestas verbales ${ }^{25}$. Se trata de un instrumento simple y fácil de usar, que no requiere ningún equipo sofisticado para ser administrado, y sus imágenes visuales son menos abstractas que las preguntas sobre la composición corporal de los niños, por lo cual fue utilizada para realizar este trabajo.

Los resultados de este estudio mostraron que la prevalencia global de la exactitud en la percepción de la imagen corporal (IMC percibido) fue del $59.4 \%$, que se refleja en un índice de concordancia aceptable de 0.30 de acuerdo con la clasificación de Landis y Koch ${ }^{31}$. El índice de concordancia de 0.30 toma sentido al mostrar diferencias entre el IMC percibido y el medido. Los niños con bajo peso tienden a sobrestimar su peso, mientras que quienes tienen peso normal, sobrepeso 
y obesidad tienden a hacer subestimaciones, particularmente en las dos últimas categorías. Montoya, et al. ${ }^{26}$ realizaron un estudio en niños hispanos de 8 a 11 años de edad en una comunidad rural de Nuevo México, EE.UU-, utilizando la CBIS, y encontraron que el $56.3 \%$ de los niños con sobrepeso y el $88.1 \%$ de los niños con obesidad subestimaron su peso corporal.

Por otra parte, la prevalencia global de la insatisfacción al comparar el IMC ideal con el medido fue del $44.4 \%$. Al comparar la discrepancia entre el IMC ideal y el medido, Montoya, et al. ${ }^{26}$ encontraron que hubo subestimaciones del $93.8 \%$ en los niños con sobrepeso y del $99 \%$ en los niños con obesidad. Estos resultados sugieren que en lugares donde la prevalencia de sobrepeso y obesidad es alta (en este estudio fue del $38 \%$, mientras que en el de Montoya, et al. ${ }^{26}$ fue del $41.7 \%$ ), los niños tienden a subestimar su peso, y lo más importante, a seleccionar imágenes ideales más delgadas que las que les corresponden. Asimismo, los resultados contrastan con los obtenidos en México en comunidades donde la prevalencia de sobrepeso y obesidad es baja, mientras que la de desnutrición es alta, dando como resultado un efecto opuesto en la selección de imágenes corporales más robustas que las que les correspondían para su peso actual21,22.

No se observó relación estadística por sexo y edad con la exactitud en el IMC percibido, pero este estuvo asociado con el IMC medido. La mayor exactitud en la selección de la imagen corporal se dio en las categorías de peso normal (86.5\%) y bajo peso $(66.7 \%)$, que contrastan con una menor magnitud en las categorías de sobrepeso (15\%) y obesidad (18.4\%), lo que remarca la subestimación que los escolares hacen de su peso a expensas de estas dos últimas categorías. Estos resultados concuerdan con los presentados en estudios realizados con la CBIS, en los que se reporta que proporciones altas de niños con sobrepeso y obesidad subestiman la percepción de su propio peso ${ }^{26,32}$.

Aunque no hubo asociación entre la discrepancia del IMC ideal con el sexo y la edad, sí la hubo con el IMC medido. La discrepancia fue absoluta en las categorías de bajo peso (100\%) y sobrepeso (100\%), y en la de obesidad $(97.3 \%)$, mientras que los niños de peso normal solo discreparon en el 5.6\%, siendo esta relación estadísticamente significativa. Dicho de otra manera, todos los niños con bajo peso seleccionaron una figura con mayor volumen que la que les correspondía, y por el contrario, todos los niños con sobrepeso y casi todos los que tenían obesidad seleccionaron figuras de menor volumen que aquella que les correspondía. Estos resultados denotan la insatisfacción mixta de los niños con su peso corporal, es decir, los niños de bajo peso tienen a seleccionar figuras de mayor volumen que las que les corresponden, al contrario de los niños con sobrepeso y obesidad. Estudios que utilizaron la CBIS muestran tendencias similares en la insatisfacción con el peso corporal, pues todas las niñas y el $88.5 \%$ de los niños con obesidad, y el $82.9 \%$ de las niñas y el 79.1 de los niños con sobrepeso, deseaban estar más delgados $^{33}$. Otros autores reportan una magnitud de insatisfacción con su peso corporal del $50 \%$ en niños entre el tercero y el sexto grados de educación bási$\mathrm{ca}^{34}$, porcentaje de insatisfacción muy similar al encontrado en este estudio (44.4\%).

Se puede concluir que los resultados de este estudio muestran que la aplicación de la CBIS en niños de la frontera del norte de México permite evaluar tanto la percepción como la insatisfacción de la imagen corporal de niños entre tercero y sexto grados, en un contexto geográfico y sociodemográfico en el que las prevalencias de sobrepeso y obesidad son altas. Asimismo, los resultados mostraron ser concordantes con los de otros estudios en los que se utilizó la CBIS en niños en edad escolar.

Falta evaluar la utilidad de la herramienta en el terreno de la salud pública en esta región del norte del país. La literatura muestra que la insatisfacción con la propia imagen corporal ha estado relacionada con diferentes eventos que emergen en población preadolescente, como baja autoestima ${ }^{34}$, trastornos alimentarios $^{35,36}$ y síntomas depresivos ${ }^{32}$. Asimismo, se ha evaluado el impacto socioeconómico y étnico en la insatisfacción con la figura corporal de niños en edad escolar ${ }^{16}$. Los resultados del presente trabajo han servido de base para el desarrollo de un estudio en curso que se realiza en colaboración con la Jurisdicción Sanitaria I, con sede en Piedras Negras, en el que se evalúa la percepción de la imagen corporal en relación con los determinantes básicos de impacto a la salud de niños de 8 y 9 años de edad (características sociodemográficas, alimentación, higiene, ejercicio y consumo de tabaco y alcohol) ${ }^{37}$ en diferentes escuelas en la ciudad.

\section{Responsabilidades éticas}

Protección de personas y animales. Los autores declaran que para esta investigación no se han realizado experimentos en seres humanos ni en animales.

Confidencialidad de los datos. Los autores declaran que han seguido los protocolos de su centro de trabajo sobre la publicación de datos de pacientes. 
Derecho a la privacidad y consentimiento informado. Los autores declaran que para tener acceso al estudio de los niños se aplicaron cartas de consentimiento informado firmadas por sus padres o tutores (ver sección de metodología).

\section{Agradecimientos}

Agradecemos ampliamente el apoyo recibido para la realización de este estudio a la Subdirección de Servicios Educativos de Piedras Negras, así como a las direcciones y las plantas docentes de las escuelas estudiadas

\section{Conflicto de intereses}

Los autores declaran no tener ningún conflicto de intereses.

\section{Bibliografía}

1. Finucane M, Stevens G, Cowan M, Danaei G, Lin J, Paciorek C, et al. National, regional and global trends in body-mass index since 1980: systematic analysis of health examination surveys and epidemiological studies with 960 country-years and 9.1 million participants. Lancet. 2011;377:557-67.

2. Ng M, Fleming T, Robinson M, Thomson B, Graetz N, Margono C, et al. Global, regional, and national prevalence of overweight and obesity in children and adults during 1980-2013: a systematic analysis for the Global Burden of Disease Study 2013. Lancet. 2014;384:766-81.

3. Instituto Nacional de Salud Pública. Encuesta Nacional de Salud y Nutrición de Medio Camino 2016. Cuernavaca, Morelos, México: INSP; 2016. p. 149.

4. Organización Mundial de la Salud. Informe de la comisión para acaba con la obesidad infantil. Ginebra: Organización Mundial de la Salud 2016. p. 50. Disponible en: http://apps.who.int/iris/bitstream/10665/ 206450/1/9789243510064_spa.pdf?Ua=1

5. Instituto Nacional de Salud Pública. Encuesta Nacional de Salud y Nutrición 2012: resultados nacionales. Cuernavaca, Morelos, México: Instituto Nacional de Salud Pública; 2012. p. 195.

6. Barquera-Cervera S, Campos-Nonato I, Rivera Dommarco JA, Belasco Bernal A. Obesidad en México: políticas y programas para su prevención. En: Rivera Dommarco JA, Hernández Ávila M, Aguilar Salinas CA, Vadillo Ortega F, Murayama Rendón C, editores. Obesidad en México: recomendaciones para una política de Estado. México: UNAM; 2012.

7. Gobierno del Estado de Coahuila. Saltillo, México. Disponible en: http:// coahuila.gob.mx/. Acceso 26 Febrero 2018.

8. Instituto Nacional de Estadística y Geografía. Encuesta Intercensal 2015. Tabulados básicos. Disponible en: http://www.beta.inegi.org.mx/proyectos/enchogares/especiales/intercensal

9. Olaiz-Fernández G, Rivera-Dommarco J, Shamah-Levy T, Rojas R, Villalpando-Hernández $S$, Hernández-Avila $M$, et al. Encuesta Nacional de Salud y Nutrición 2006. Cuernavaca, México: Instituto Nacional de Salud Pública; 2006. p. 131.

10. Instituto Nacional de Salud Pública. Encuesta Nacional de Salud y Nutrición 2012. Resultados por entidad federativa, Coahuila. Cuernavaca, México: INSP; 2013. p. 111

11. Alonso-Tovar R, Uribe-Salas FJ. Evaluación del estado nutricional en niños en edad escolar en Piedras Negras, Coahuila, 2016: un estudio piloto. Rev Med de Torreón. 2017;9:25-31.

12. Anaya G, Al-Delaimy WK. Effect of the US-Mexico border region in cardiovascular mortality: ecological time trend analysis of Mexican border and no border municipalities from 1998 to 2012. BMC Public Health. 2017:17:400.
13. Truby H, Paxton SJ. Development of the children's body image scale. $\mathrm{Br}$ J Clin Psychol. 2002;41:185-203.

14. Field AE, Cheung L, Wolf AM, Herzog DB, Gortmaker SL, Colditz GA Exposure to the mass media and weight concerns among girls. Pediatrics. 1999;103:E36.

15. Field AE, Austin SB, Camargo CA, Taylor CB, Striegel-Moore RH, Loud KJ, et al. Exposure to the mass media, body shape concerns, and use of supplements to improve weight and shape among male and female adolescents. Pediatrics. 2005;116:e214-20.

16. Robinson TN, Chang JY, Haydel KF, Killen JD. Overweight concerns and body dissatisfaction among third-grade children: the impacts of ethnicity and socioeconomic status. J Pediatrics. 2001;138:181-7.

17. Leppers I, Tiemeier H, Swanson SA, Verhulst FC, Jaddoe VWV Franco $\mathrm{OH}$, et al. Agreement between weight status and perceived body size and the association with body size satisfaction in children. Obesity (Silver Spring). 2017;25:1956-64.

18. Byrne NM, Hills AP. Should body image scales designed for adults be used with adolescents? Percept Mot Skills. 1996;82:747-53

19. Fallon $A E$, Rozin P. Sex differences in perceptions of desirable body shape. J Abnorm Psychol. 1985;94:102-5.

20. Collins ME. Body figure perceptions and preferences among preadolescent children. Int J Eat Disord.1991;10:199-208.

21. Cortes-Martínez G, Vallejo-de la Cruz NL, Pérez-Salgado D, Ortiz-Hernández L. Utilidad de siluetas corporales en la evaluación del estado nutricional en escolares y adolescentes de la Ciudad de México. Bol Med Hosp Infant Mex. 2009;66:511-21.

22. Oliva-Peña $Y$, Ordóñez-Luna $M$, Santana-Carvajal $A$, Marín-Cárdenas $A D$, Pech GA, Castillo IAG. Concordancia del IMC y la percepción de la imagen corporal en adolescentes de una localidad suburbana de Yucatán. Rev Biomed. 2016;27:49-60.

23. Montoya Resio Y, García Maldonado AM. Percepción de la imagen corporal de niños y niñas de 9 a 12 años de la zona escolar P239, del Municipio de Zacualpan, Estado de México en 2013. [Tesis]. Toluca, México: Facultad de Medicina, Universidad Autónoma del Estado de México; 2013.

24. Saxton J, Hill C, Chadwick P, Wardle J. Weight status and perceived body size in children. Arch Dis Child. 2009;94:944-9.

25. Hussin DK, Mohamad IH, Al-Hamad HA, Makboul G, Elshazly M. Weight status and perceived body size image in overweight and obese children 8-12 years old. Alexandria J Med. 2011;47:365-71.

26. Montoya $\mathrm{C}$, Boursaw $\mathrm{B}$, Tigges $\mathrm{B}$, Lobo $\mathrm{M}$. Mirror, mirror on the wall: children's preferences and self-perception of weight in a rural Hispanic community. J Pediatr Health Care. 2016;30:528-34.

27. International Society for the Advancement of Kinanthropometry (ISAK) International standards for anthropometric assessment. Potchefstroom, South Africa: ISAK; 2001. p.133.

28. Truby H, Paxton SJ. The Children's Body Image Scale: reliability and use with international standards for body mass index. $\mathrm{Br} \mathrm{J}$ Clin Psychol. 2008;47:119-24

29. Romero F. Tablas de percentiles de peso, talla y BMI. Programa para Windows. 2009. Disponible en: https://www.ac.uma.es/ felipe/bmi

30. Zklo M, Nieto J. Epidemiology. Beyond the basics. Gaithersburg: Aspen Publishers Inc.; 2000.

31. Landis JR, Koch GG. The measurement of observer agreement for categorical data. Biometrics. 1977;33:159-74.

32. Gray MN, Crawford MJ, Follansbee-Junger K, Dumont-Driscoll MC, Janicke DM. Association between actual and perceived weight and psychosocial functioning in children: the importance of child perceptions. Child Obes. 2012;8:147-54.

33. Knowles G, Ling FCM, Thomas GN, Adab P, McManus AM. Body size dissatisfaction among young Chinese children in Hong Kong: a cross-sectional study. Public Health Nutr. 2015;18:1067-74.

34. Paxton SJ, Neumark-Sztainer D, Hannan PJ, Eisenberg ME. Body dissatisfaction prospectively predicts depressive mood and low self-esteem in adolescent girls and boys. J Clin Child Adolesc Psychol. 2006;35:539-49.

35. Schur EA, Sanders M, Steiner H. Body dissatisfaction and dieting in young children. Int J Eat Disord. 2000;27:74-82.

36. Killen JD, Taylor CB, Hayward C, Haydel KF, Wilson DM, Hammer L, et al. Weight concerns influence the development of eating disorders: a 4-year prospective study. J Consult Clin Psychol. 1996;64:936-40.

37. Secretaría de Salud. Resultados de la construcción de la basal para el Sistema de Monitoreo de Determinantes de la Salud, en niños y niñas de 8 y 9 años de edad en México, 2010-2011. México: Dirección General de Promoción de la Salud 2011. 